VOL. 28 (1983), 91-99.

\title{
BEURLING'S ORDINARY VALUE
}

\section{Shinji Yamashita}

Let $n(w, f)$ be the number of $w$-points of $f$ meromorphic in $D=\{|z|<1\}$. Beurling defined the quantity $\bar{n}(\omega, f)$ and called $w$ an ordinary value of $f$ if $\bar{n}(\omega, f)<\infty$. We shall consider the intermediate quantity $\underline{n}(w, f)$ in the sense that $n(w, f) \leq \underline{n}(w, f) \leq \bar{n}(w, f)$, and construct two bounded holomorphic functions $f_{1}$ and $f_{2}$ of finite Dirichlet integrals in $D$ for which

$$
0=n\left(0, f_{1}\right)<\underline{n}\left(0, f_{1}\right)<\vec{n}\left(0, f_{1}\right)<\infty
$$

and

$$
0=n\left(0, f_{2}\right)<\underline{n}\left(0, f_{2}\right)<\bar{n}\left(0, f_{2}\right)=\infty
$$

\section{Introduction}

Let $W$ be the Riemann sphere of radius $\frac{1}{2}$ touching the complex plane $C$ at 0 . The sphere $W$ is endowed with the chordal distance $X(\cdot, \cdot)$ and with the element of the spherical area $d w(w)$ at $w \in W$, being expressed as $d \omega(\omega)=\left(1+|w|^{2}\right)^{-2} d x d y$, if $w \neq \infty$ is identified with its projection $x+i y \in \mathbb{C}$. Then the area of the Riemannian image of $D=\{|z|<1\}$ by $f$ meromorphic in $D$ over the spherical cap

$$
C(a, r)=\{w \in W ; X(w, a)<r\} \quad(a \in W, 0<r \leq 1)
$$

Received 14 April 1983. 


$$
A(a, r, f)=\iint_{C(a, r)} n(w, f) d w(w),
$$

where $n(w, f)$ is the number of the zeros of $f-w$ in $D$, the order being counted. We then set, for $a \in W$,

$$
\begin{aligned}
& \bar{n}(\alpha, f)=\underset{r \rightarrow 0}{\lim \sup }\left(\pi r^{2}\right)^{-1} A(a, r, f), \\
& \underline{n}(a, f)=\underset{r \rightarrow 0}{\liminf \left(\pi r^{2}\right)^{-1} A(a, r, f) ;}
\end{aligned}
$$

here

$$
\pi r^{2}=\iint_{C(a, r)} d w(w)
$$

It follows from the lower semicontinuity of $n(w, f)$ that

$$
n(a, f) \leq \underline{n}(a, f) \leq \bar{n}(a, f)
$$

at each $a \in W$. If $\bar{n}(a, f)<\infty(n(a, f)<\infty$, respectively $)$, then $a$ is called an ordinary value (a lower ordinary value, respectively) of $f$; the definition of ordinary value is due to Beurling [1, p. 11]. Furthermore, if

$$
\iint_{D}\left(\left|f^{\prime}(z)\right| /\left(1+|f(z)|^{2}\right)\right)^{2} d x d y=A(0,1, f)<\infty,
$$

where $z=x+i y$, then

$$
n(a, f)=\underline{n}(a, f)=\bar{n}(a, f)<\infty
$$

for $d w$-almost every $a \in W ;$ see [2, Theorem 6.3 on p. 118, and the inequality at line 11 from below on p. 149].

$$
\begin{array}{r}
\text { Now, if } n(a, f)=\infty \text {, then, apparently, } \\
\qquad \underline{n}(a, f)=\bar{n}(a, f)=\infty
\end{array}
$$

without the assumption (1.1). Does the equaltiy

$$
\underline{n}(a, f)=\bar{n}(a, f) \quad(\text { possibly equal to } \infty)
$$

hold if $n(a, f)<\infty$ for $f$ satisfying (1.1)?

We shall construct two examples which answer this question in the negative. 
REMARK. An obvious example erases the doubt that $n(a, f)<\infty$ for each $a \in W$ if (1.1) is satisfied.

THEOREM 1. There exists a bounded univalent holomorphic function $f$ in $D$, satisfying $(1.1)$ and $)$

$$
0=n(0, f)<\underline{n}(0, f)<\bar{n}(0, f)<\infty .
$$

We note that if $f$ is bounded in $D$, then (I.I) is equivalent to

$$
\iint_{D}\left|f^{\prime}(z)\right|^{2} d x d y<\infty \quad(z=x+i y) .
$$

The proof of Theorem 1 is rather easy in contrast with that of

THEOREM 2. There exists a bounded holomorphic function $f$ in $D$, satisfying (1.1) and

$$
0=n(0, f)<\underline{n}(0, f)<\bar{n}(0, f)=\infty .
$$

We note that 0 is a lower ordinary value yet not an ordinary value of $f$ in Theorem 2 .

\section{Proof of Theorem 1}

First of all, $\arg w$ of $w \in W-\{0, \infty\}$ means that of the projection of $w$ into $\mathbb{C}$. Letting $a_{k}=2^{-k}, k=1,2, \ldots$, we consider the simply connected domain $S$ over $W$ defined by $\dot{s}=\left\{w \in C\left(0, a_{2}\right) ;-\pi / 2<\arg w<0\right\}$

$$
\cup \underset{n=1}{\infty}\left\{w \in c\left(0, a_{2 n}\right)-\bar{c}\left(0, a_{2 n+1}\right) ; 0 \leq \arg w<\pi / 2\right\} .
$$

Let $f$ be a one-to-one conformal mapping from $D$ onto $S$, which may be considered as a bounded holomorphic function satisfying (1.1).

$$
\text { Let } r=2^{-t}, 2 n \leq t<2 n+1 \text {, so that } a_{2 n+1}<r \leq a_{2 n}
$$

$(n=1,2, \ldots)$. Then

$$
A(0, r, f)=3 \pi r^{2}+I_{n}+\frac{1}{4} \pi\left(r^{2}-a_{2 n+1}^{2}\right) \text {, }
$$

where 


$$
I_{n}=\sum_{k=n+1}^{\infty} \frac{1}{4} \pi\left(a_{2 k}^{2}-a_{2 k+1}^{2}\right)=\frac{\pi}{5} \cdot \frac{1}{16^{n+1}} .
$$

Therefore

$$
\left(\pi r^{2}\right)^{-1} A(0, r, f)=\frac{1}{2}-\frac{4}{5} \cdot \frac{4^{t}}{16^{n+1}}
$$

and

$$
3 / 10<\left(\pi r^{2}\right)^{-1} A(0, r, f) \leq 9 / 20 \text {. }
$$

Let $r=2^{-t}, 2 n+1 \leq t<2 n+2$, so that $a_{2 n+2}<r \leq a_{2 n+1}$ $(n=1,2, \ldots)$. Then

$$
A(0, r, f)=\frac{1}{4} \pi r^{2}+I_{n},
$$

so that

$$
\left(\pi r^{2}\right)^{-1} A(0, r, f)=\frac{1}{4}+\frac{1}{5} \cdot \frac{4^{t}}{16^{n+1}},
$$

together with

$$
3 / 10 \leq\left(\pi r^{2}\right)^{-1} A(0, r, f)<9 / 20 .
$$

It now follows from (2.1) and (2.2) that

$$
3 / 10=\underline{n}(0, f)<\bar{n}(0, f)=9 / 20,
$$

whence follows (1.2).

\section{Proof of Theorem 2}

We shall make use of the following

LEMMA. Given $A>0, B>0$, and $s>0$, there exist a natural number $N$ and a positive number $\lambda<s$ such that

$$
\pi N\left[A^{2}-(A-\lambda)^{2}\right]=B
$$

The proof is elementary and is omitted.

To prove Theorem 2 we choose a pair $\left\{p_{m}\right\}_{m=1}^{\infty}$ and $\left\{q_{m}\right\}_{m=1}^{\infty}$ of sequences of natural numbers inductively as follows. First, let $p_{1}=2$. 
Then, given $p_{m}(m \geq 1)$ we select $q_{m}>p_{m}$ such that $q_{m}^{-\frac{1}{2}}<2^{-p_{m}}$. We choose then $p_{m+1}>q_{m}$ such that $2^{-p_{m+1}}<q_{m}^{-\frac{1}{2}}$.

We set $a_{m}=2^{-p}$ and $b_{m}=q_{m}^{-\frac{1}{2}}(m=1,2, \ldots)$, so that

$$
1 / 4=a_{1}>b_{1}>a_{2}>\ldots>a_{m}>b_{m}>a_{m+1}>\ldots+0
$$

It then follows from the lemma that there exist a natural number $\nu_{m}$ and a positive number $\varepsilon_{m}<a_{m}-b_{m}$ such that

$$
\pi \nu_{m}\left[a_{m}^{2}-\left(a_{m}-\varepsilon_{m}\right)^{2}\right]=p_{m}^{-2} \quad(m=1,2, \ldots) \text {. }
$$

We notice that $v_{m}^{-1} p_{m}^{-2}$ is the area of the spherical ring $R\left(a_{m}\right)=c\left(0, a_{m}\right)-\bar{C}\left(0, a_{m}-\varepsilon_{m}\right) \quad(m=1,2, \ldots)$. It also follows from the lemma that there exist a natural number $\mu_{m}$ and a positive number $\delta_{m}<b_{m}-a_{m+1}$ such that

$$
\pi \mu_{m}\left[b_{m}^{2}-\left(b_{m}-\delta_{m}\right)^{2}\right]=2^{-q} \quad(m=1,2, \ldots)
$$

In the present case, $\mu_{m}^{-1} 2^{-q} m$ is the area of the spherical ring $R\left(b_{m}\right)=c\left(0, b_{m}\right)-\bar{c}\left(0, b_{m}-\delta_{m}\right) \quad(m=1,2, \ldots)$.

Let $S_{1}$ be the Riemann surface over $W$, in the form of a ribbon, which winds its way just $\nu_{m}$ times over $R\left(a_{m}\right)$, and just $\mu_{m}$ times over $R\left(b_{m}\right) \quad(m=1,2, \ldots)$, and which tends to the origin; see Figure 1 where the case $v_{m}=\mu_{m}=1 \quad(m=1,2, \ldots)$ is expressed. More precisely, $S_{1}$ covers

$$
c\left(0, a_{1}\right)-\bigcup_{m=1}^{\infty}\left[R\left(a_{m}\right) \cup R\left(b_{m}\right)\right]
$$

once by the parts which we shall call bridges, while $S_{1}$ covers $R\left(a_{m}\right)$ 


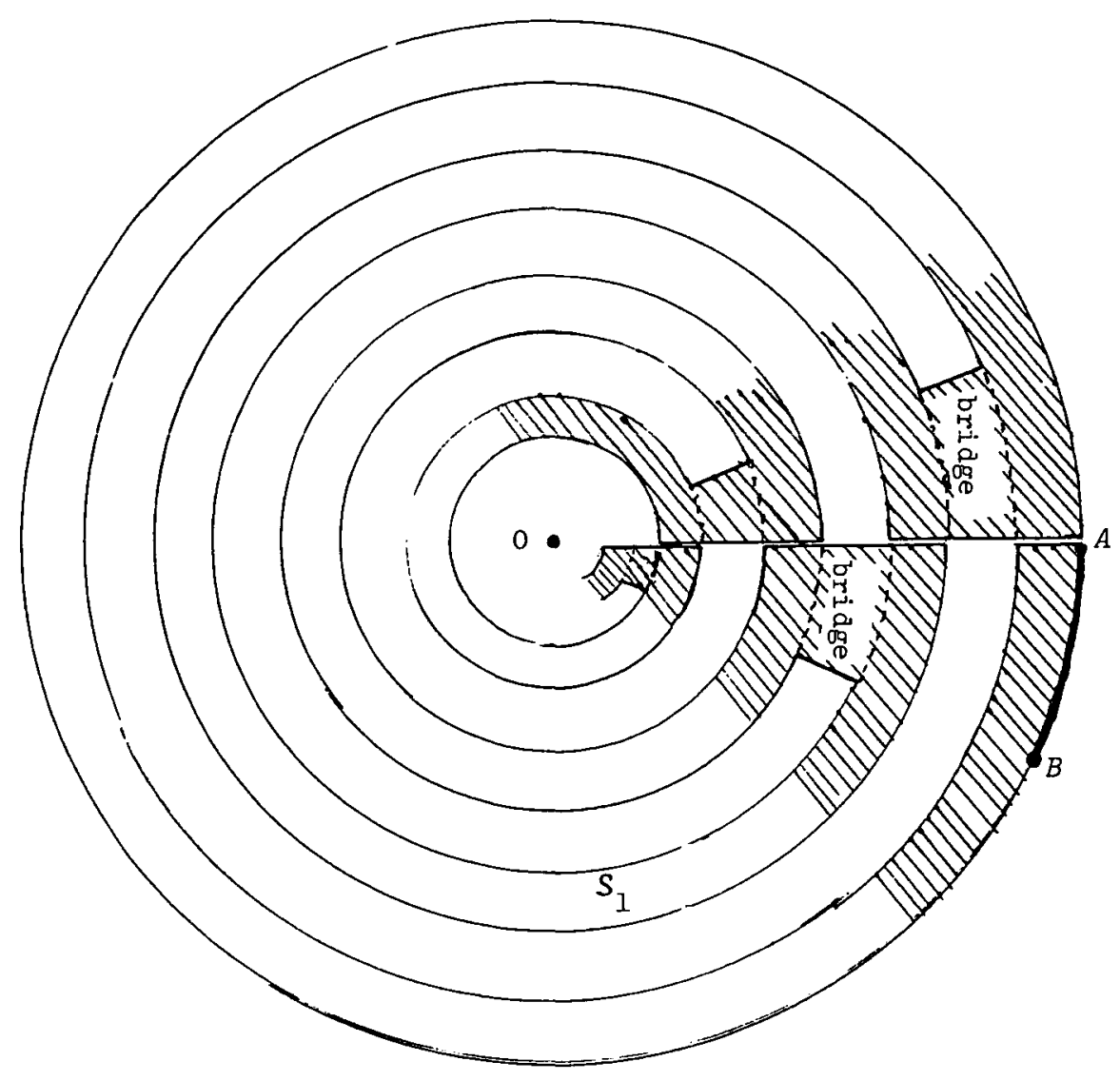

FIGURE 1 
$\left(R\left(b_{m}\right)\right.$ respectively $)$ just $\nu_{m}\left(\mu_{m}\right.$ respectively) times except for a cross cut of $R\left(a_{m}\right) \quad\left(R\left(b_{m}\right)\right.$ respectively) which $S_{1}$ covers just $\nu_{m}-1$ $\left(\mu_{m}-1\right.$ respectively $)$ times $(m=1,2, \ldots)$.

Let $S_{2}$ be a one-sheeted ribbon over $C(0,1 / 3)$ such that $S_{2}$ ends at 0 in the form

$$
S_{2} \cap C\left(0, r_{0}\right)=\left\{w \in C\left(0, r_{0}\right) ;|\arg w|<\pi / 2\right\}
$$

for a certain $r_{0}>0$; see Figure 2. We then paste $S_{1}$ and $S_{2}$ along the circular arc $A B$ to obtain the resulting simply connected Riemann surface $S$ over $W$. Let $f$ be a one-to-one conformal mapping from $D$ onto $S$, which we may consider as a bounded holomorphic function in $D$.

We first consider the sequence $a_{m} \downarrow 0$. Then

$$
A\left(0, a_{m}, f\right) \geq \text { the area of } s_{1} \text { over } R\left(a_{m}\right)=p_{m}^{-2}
$$

so that

$$
\left(\pi a_{m}^{2}\right)^{-1} A\left(0, a_{m}, f\right) \geq \pi^{-1}{ }^{2 p} m_{p_{m}^{-2}} \rightarrow \infty,
$$

whence $\bar{n}(0, f)=\infty$.

We next observe that, for each sequence $r_{n}+0(n \geq 1)$, the following holds for $r_{n}<r_{0}$;

$$
\begin{aligned}
A\left(0, r_{n}, f\right) & \geq \text { the area of. the part of } S_{2} \text { over } c\left(0, r_{n}\right) \\
& =\frac{1}{2} \pi r_{n}^{2},
\end{aligned}
$$

so that $\underline{n}(0, f) \geq \frac{1}{2}$.

To prove $\underline{n}(0, f)<\infty$ we consider the sequence $b_{m}+0$. Then

(3.1) $A\left(0, b_{m}, f\right)=$ the area of the bridges over $c\left(0, b_{m}\right)+$ the area of

$$
\text { the part of } S_{2} \text { over } c\left(0, b_{m}\right)+\sum_{k=m+1}^{\infty} p_{k}^{-2}+\sum_{k=m}^{\infty} 2^{-q_{k}} \text {, }
$$

so that 


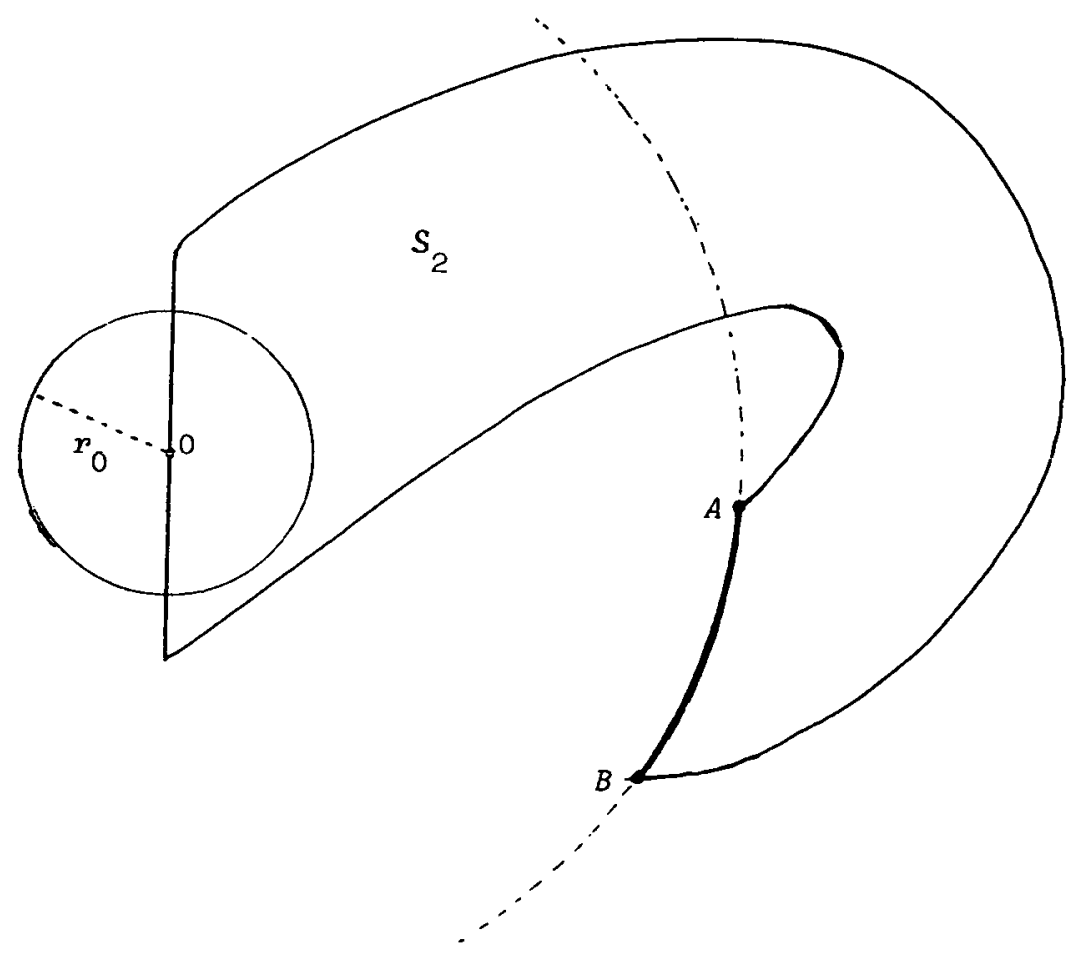

FIGURE 2 
the third term + the fourth term of (3.1)

$$
\begin{aligned}
& \leq \sum_{k=p_{m+1}}^{\infty} k^{-2}+\sum_{k=q_{m}}^{\infty} 2^{-k} \leq \sum_{k=p_{m+1}}^{\infty}\left(\frac{1}{k-1}-\frac{1}{k}\right)+2^{1-q_{m}} \\
& =\frac{1}{p_{m+1}-1}+2^{1-q_{m}} .
\end{aligned}
$$

Since the first term of (3.1) is less than $\pi b_{m}^{2}$, it follows that

$$
\left(\pi b_{m}^{2}\right)^{-1} A\left(0, b_{m}, f\right) \leq 1+\frac{1}{2}+\pi^{-1}+\pi^{-1} q_{m}^{2}{ }^{1-q} m
$$

for $b_{m}<r_{0}$, because of ${ }^{\prime} q_{m} \leq p_{m+1}-1$. Letting $m \rightarrow \infty$ one observes that $\underline{n}(0, f) \leq 3 / 2+\pi^{-1}$.

Since

$$
\sum_{m=1}^{\infty} p_{m}^{-2}+\sum_{m=1}^{\infty} 2^{-q_{m}} \leq \sum_{k=p_{1}}^{\infty} k^{-2}+\sum_{k=q_{1}}^{\infty} 2^{-k} \leq \frac{1}{p_{1}-1}+2^{1-q_{1}}
$$

it is easy to observe that $f$ satisfies (I.I).

\section{References}

[1] Arne Beurling, "Ensembles exceptionnels", Acta Math. 72 (1940), 1-13.

[2] Stanislaw Saks, Theory of the integral, second revised edition (translated by L.C. Young. Monografie Matematyczne, 7. Hafner, New York, 1937).

\section{Department of Mathematics,}

Tokyo Metropolitan University,

Fukazawa 2-1-1, Setagaya-ku,

Tokyo,

158 Japan. 